\title{
Pneumomediastinum: A Rare Complication of Endobronchial Ultrasound Guided Fine Needle Aspiration
}

\section{Biplab K. Saha}

Department of Pulmonary and Critical Care Medicine, Ozarks Medical Center, West Plains, USA

Received November 9, 2020; Accepted January 31, 2022.

Key words: EBUS-TBNA - Pneumomediastinum - Complications

Abstract: Endobronchial ultrasound guided transbronchial needle aspiration (EBUS-TBNA) is a commonly performed outpatient procedure used for the diagnosis, staging of lung cancer, and the evaluation of thoracic lymphadenopathy of unknown origin. With the advent of this minimally invasive technology, mediastinoscopy, once the gold standard, has fallen out of favour. Pneumomediastinum is a rare complication of EBUS-TBNA and can often be managed conservatively. We present a case of a 52-year-old female who developed pneumomediastinum following EBUS-TBNA and improved with expectant management in the emergency department. We discuss the proposed pathophysiology of this rare occurrence that usually follows a benign course. Severe complications, such as mediastinitis and tracheal tear, need to be excluded promptly.

Mailing Address: Biplab K. Saha, MD., Ozarks Medical Center, 1100 Kentucky Avenue, West Plains, Missouri 65775, USA; e-mail: spanophiliac@yahoo.com 


\section{Introduction}

Pneumomediastinum is a commonly encountered medical condition in the emergency department (ED). It can occur in isolation or be associated with pneumothorax. Common causes of pneumomediastinum are blunt force trauma to the chest, obstructive pulmonary diseases, and mediastinitis, which occurs most frequently as an iatrogenic complication of upper aero-digestive tract procedures. Endobronchial ultrasound guided transbronchial needle aspiration (EBUS-TBNA) refers to the performance of fine needle aspiration of the target lesion under direct ultrasound visualization during bronchoscopy. EBUS-TBNA is currently the procedure of choice for diagnosis and staging of lung cancer due to the minimal invasiveness (Vyas et al., 2013). EBUS-TBNA allows sampling of most hilar and mediastinal lymph nodes except paraaortic and paraesophageal lymph node stations (stations 5, 6, 8, and 9). However, simultaneous endoscopic ultrasound using EBUS scope (EUS-B) enables the operator to access the lower mediastinal lymph node stations (stations 8 and 9 ) by a transesophageal approach. EUS-B guided fine needle aspiration (EUS-NA) provides a more complete staging of the mediastinum with increased sensitivity and negative predictive value (Bugalho et al., 2018). The procedure is usually performed as an outpatient with minimal risk of complications. Pneumomediastinum is a rare complication of EBUS-TBNA that has been reported in the form of case reports in the literature. The pathophysiology behind the causation of pneumomediastinum is unclear, and most patients follow a benign trajectory. We report the rare occurrence of pneumomediastinum in a patient following EBUS-TBNA, who improved with conservative management. The proposed pathophysiology and management are also discussed.

\section{Case report}

A 52-year-old female presented to the hospital with intermittent anterior chest pain and self-reported episodes of hemoptysis of several months duration. The patient was an active smoker with more than thirty-five pack-year history of smoking. She was treated with antibiotics multiple times in the recent past for recurrent pneumonia. On admission, her vital signs were stable. Physical examination was unremarkable. She underwent a computed tomographic angiogram of the chest to rule out pulmonary embolism. Computed tomography $(\mathrm{CT})$ of the chest revealed hilar and mediastinal lymphadenopathy, and concerns were raised regarding the possibility of lymphoma or a primary lung malignancy. The patient underwent EBUS-TBNA of right lower paratracheal (4R), subcarinal (7) and right hilar (10R) lymph node stations. The procedure was performed under general anesthesia using a $21 \mathrm{~g}$ needle. Low tidal volume ventilation with positive end-expiratory positive pressure (PEEP) of $5 \mathrm{~cm}$ of water was used throughout the procedure. Care was taken to avoid the development of auto-PEEP. There was no endobronchial lesion, and the TBNA was uneventful. There was minimal bleeding. The patient was discharged home in a stable condition following the procedure. However, the patient 


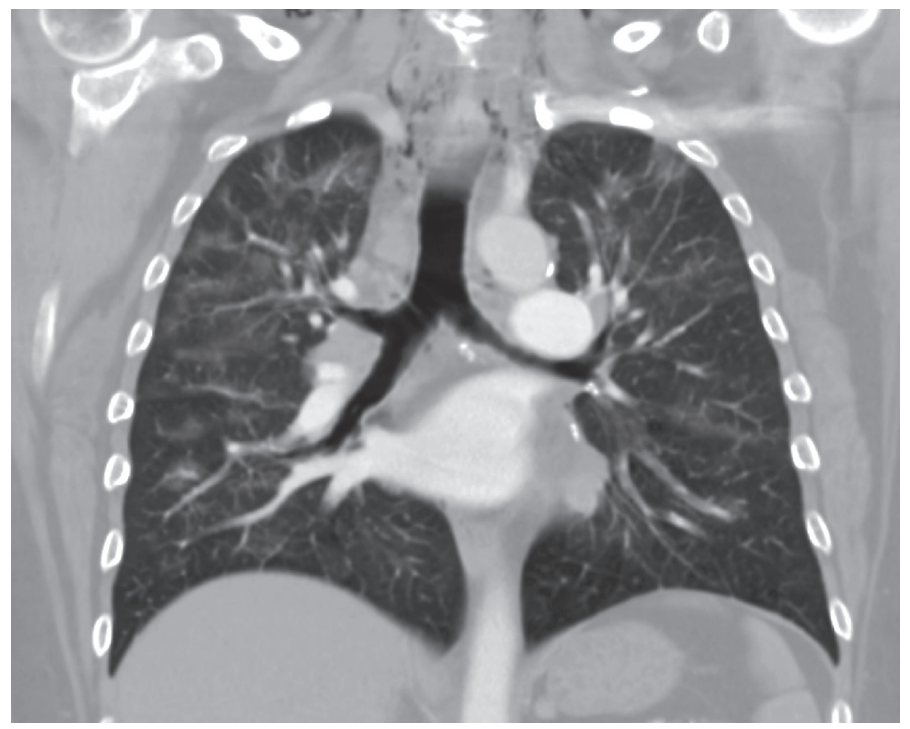

Figure 1 - Coronal computed tomography scan of the chest revealing pneumomediastinum with extension at the base of the neck.

presented to the ED that night with worsening chest pain and difficulty breathing. Her vital signs on presentation were a blood pressure of 147/88 mm Hg, a pulse rate of 93 beats per minute, a temperature of $36.83^{\circ} \mathrm{C}$, a respiratory rate of 17 breaths per minute, and oxygen saturation of $96 \%$ with the patient breathing ambient air. Physical examination revealed a non-toxic patient in no acute distress. The remainder of the physical examination, including chest auscultation, was completely normal. A chest X-ray was unremarkable. Representative slides of the CT scan of the chest are shown in Figures 1 and 2. The coronal (Figure 1) and axial (Figure 2) views revealed pneumomediastinum with an extension of the air up to the base of the neck. There was no evidence of mediastinitis on transverse imaging. A gastrografin esophagram revealed no esophageal perforation. The patient was
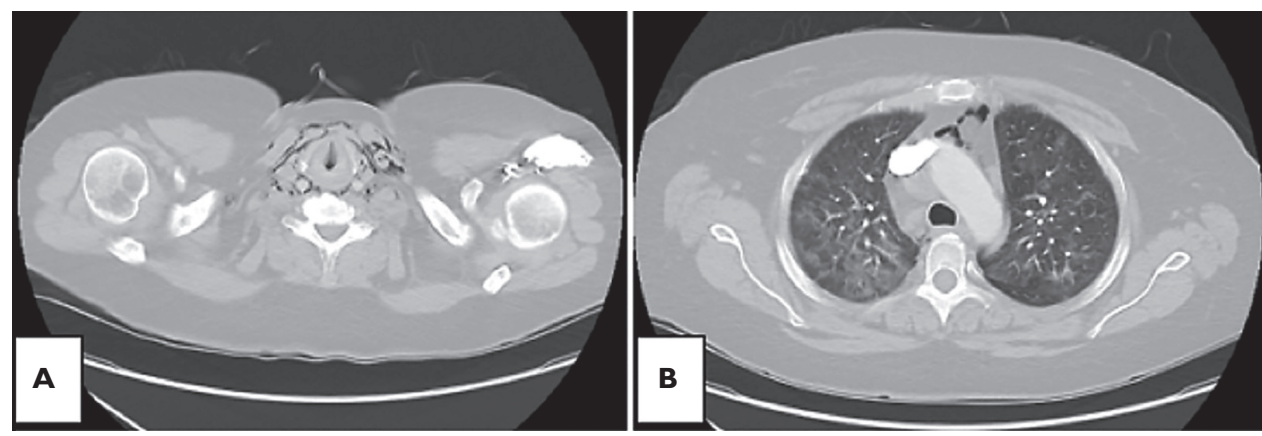

Figure 2 - Axial computed tomography scan on the chest at the level of the vocal cords (A) and upper trachea (B) showing varying degrees of air around the airways and vascular structures. 
treated with pain medication with subsequent improvement of her symptoms. She was discharged home from the ED with a planned follow-up with the pulmonologist. The lymph node fine needle aspirations (FNAs) were negative for malignancy.

\section{Discussion}

This case represents a rare complication of EBUS-TBNA. EBUS-TBNA has revolutionized lung cancer diagnosis and simultaneous staging. It is a sensitive, safe, and less invasive procedure compared to mediastinoscopy and has largely replaced the later in the diagnostic algorithm of lung cancer. Complications from EBUS-TBNA are rare and mostly involve mild bleeding (Asano et al., 2013). Only a handful of cases of isolated pneumomediastinum have been reported in the literature, in the form of case reports (Piroddi et al., 2017). A review of all published trials of EBUS-TBNA between 1995-2012 did not show any case of pneumomediastinum (von Bartheld et al., 2014). Similarly, in a nationwide study from Japan, there was no report of pneumomediastinum (Asano et al., 2013). The exact pathophysiologic mechanism of pneumomediastinum following TBNA is unclear. One possible explanation is the Macklin effect. Macklin effect refers to the development of pneumomediastinum due to increased intrathoracic pressure. The increased intrathoracic pressure leads to alveolar rupture and forceful expulsion of the alveolar air in the mediastinal space following centripetal dissection along the bronchovascular bundle (Macklin and Macklin, 1944). Pneumomediastinum can be seen in patients with blunt force trauma to the chest, following forceful coughing or sneezing, and in the setting obstructive pulmonary diseases. This can occur with or without accompanying pneumothorax.

EBUS-TBNA utilizes the application of small caliber needles for the purpose of lymph node sampling under direct ultrasound visualization. The procedure is generally safe and well-tolerated by patients. The risk of pneumothorax following EBUS-TBNA has been reported to be $0.03 \%$ (Asano et al., 2013). Significant trauma to the airways leading to leakage of air is therefore highly unlikely. The etiology of the pneumomediastinum in our case is unclear. The patient underwent the procedure under general anesthesia with low tidal volume ventilation and low PEEP, thus, making barotrauma an unlikely causative etiology. Similarly, there was no coughing, breath stacking, or auto-PEEP generation, which could have increased the intrathoracic pressure. As a result, it is plausible that in very rare occasions, FNA during EBUS can in fact cause isolated pneumomediastinum by the creation of temporary and self-limited tracheomediastinal or bronchomediastinal fistula.

Pneumomediastinum following TBNA should be managed conservatively. However, it is important to rule out mediastinitis, as this can be rapidly fatal in the absence of prompt intervention (Pastene et al., 2020). Whether pneumomediastinum represents a rare or a relatively underdiagnosed complication due to lack of significant symptoms - is unknown. Patients might not seek medical attention due to mild chest discomfort following bronchoscopy, which could be 
the sole manifestation of pneumomediastinum. In addition, chest X-ray might be relatively insensitive to identify mild cases, as was the case in our patient (Zylak et al., 2000). Knowledge of this unusual complication is crucial for clinicians to prevent unnecessary surgical interventions, such as chest tube insertion. At the same time, careful evaluation is necessary to exclude serious damage to the upper airway structure, such as esophageal perforation and tracheal tear. Bronchomediastinal fistula and mediastinitis following EBUS-TBNA have been reported (Bougioukas et al., 2019).

\section{References}

Asano, F., Aoe, M., Ohsaki, Y., Okada, Y., Sasada, S., Sato, S., Suzuki, E., Semba, H., Fukuoka, K., Fujino, S., Ohmori, K. (2013) Complications associated with endobronchial ultrasound-guided transbronchial needle aspiration: A nationwide survey by the Japan Society for Respiratory Endoscopy. Respir. Res. 14(1), 50.

Bougioukas, I., Seipelt, R., Huwer, H. (2019) Bronchial fistula and pneumomediastinum after EBUS-TBNA following mediastinoscopy. Thorac. Cardiovasc. Surg. Rep. 8(1), e11-e13.

Bugalho, A., de Santis, M., Szlubowski, A., Rozman, A., Eberhardt, R. (2018) Trans-esophageal endobronchial ultrasound-guided needle aspiration (EUS-B-NA): A road map for the chest physician. Pulmonology 24(1), $32-41$.

Macklin, M. T., Macklin, C. C. (1944) Malignant interstitial emphysema of the lungs and mediastinum as an important occult complication in many respiratory diseases and other conditions: An interpretation of the clinical literature in the light of laboratory experiment. Medicine 23(4), 281-358.

Pastene, B., Cassir, N., Tankel, J., Einav, S., Fournier, P.-E., Thomas, P., Leone, M. (2020) Mediastinitis in the intensive care unit patient: A narrative review. Clin. Microbiol. Infect. 26(1), 26-34.

Piroddi, I. M. G., Gatto, P., Perazzo, A., Barlascini, C., Nicolini, A. (2017) Pneumomediastinum following endobronchial ultrasound-guided transbronchial needle aspiration: A case report. Tanaffos 16(3), 245-247.

von Bartheld, M. B., van Breda, A., Annema, J. T. (2014) Complication rate of endosonography (endobronchial and endoscopic ultrasound): A systematic review. Respiration 87(4), 343-351.

Vyas, K. S., Davenport, D. L., Ferraris, V. A., Saha, S. P. (2013) Mediastinoscopy: Trends and practice patterns in the United States. South. Med. J. 106(10), 539-544.

Zylak, C. M., Standen, J. R., Barnes, G. R., Zylak, C. J. (2000) Pneumomediastinum revisited. Radiographics 20(4), 1043-1057. 